\title{
Use of Green Fluorescent Protein Color Variants Expressed on Stable Broad-Host-Range Vectors to Visualize Rhizobia Interacting with Plants
}

\author{
Nico Stuurman, Cristina Pacios Bras, Helmi R. M. Schlaman, André H. M. Wijfjes, \\ Guido Bloemberg, and Herman P. Spaink \\ Leiden University, Institute of Molecular Plant Sciences, Wassenaarseweg 64, 2333 AL Leiden, The Netherlands. \\ Accepted 20 July 2000.
}

\begin{abstract}
We developed two sets of broad-host-range vectors that drive expression of the green fluorescent protein (GFP) or color variants thereof (henceforth collectively called autofluorescent proteins [AFPs]) from the lac promoter. These two sets are based on different replicons that are maintained in a stable fashion in Escherichia coli and rhizobia. Using specific filter sets or a dedicated confocal laser scanning microscope setup in which emitted light is split into its color components through a prism, we were able to unambiguously identify bacteria expressing enhanced cyan fluorescent protein (ECFP) or enhanced yellow fluorescent protein (EYFP) in mixtures of the two. Clearly, these vectors will be valuable tools for competition, cohabitation, and rescue studies and will also allow the visualization of interactions between genetically marked bacteria in vivo. Here, we used these vectors to visualize the interaction between rhizobia and plants. Specifically, we found that progeny from different rhizobia can be found in the same nodule or even in the same infection thread. We also visualized movements of bacteroids within plant nodule cells.
\end{abstract}

The symbiotic interaction between rhizobia and leguminous plants is often very host specific, i.e., certain bacterial species interact with only one or a few plant species (Hadri et al. 1998). For instance, Rhizobium leguminosarum biovar trifolii interacts only with plants from the Trifolium genus but not with other leguminous plant genera (Hadri et al. 1998). In other cases, one bacterial species can interact with many different plant species, e.g., Rhizobium sp. strain NGR234 and Sinorhizobium fredii strain USDA257 which nodulate on many genera of subtropical and tropical legumes (Pueppke and Broughton 1999). Competition studies are very useful tools to establish the relative 'fitness' of a certain bacterial strain to infect a specific plant species. However, competition is difficult to monitor and often requires reisolation of bacteria from nodules followed by cumbersome identification of the species present (Vincent 1970). We therefore sought a more direct

Corresponding author: Nico Stuurman; Telephone: +31 71527 4909; Fax: +31 71527 4999; E-mail: stuurman@ rulbim.leidenuniv.nl method to continuously monitor the individual strains of bacteria within a mixture.

Recent years have seen the advent of the use of the green fluorescent protein (GFP) in biological studies. Since no cofactors are needed for the folded GFP protein to develop fluorescence, it has been possible to use the gene encoding GFP as a marker in many different organisms (Chalfie et al. 1994; Matus 1999). For instance, GFP has successfully been used to follow various aspects of the nodulation process after expression in rhizobia (Cheng and Walker 1998; Gage et al. 1996; Xi et al. 1999). By screening and by rational design, either together or separately, a number of GFP mutants have been derived that display altered excitation and emission properties (Tsien 1998). These GFP color variants (collectively called autofluorescent proteins [AFPs]) allow the simultaneous expression of multiple fluorescent markers and-using proper ways to excite the samples and detect the emitted light-they can easily be distinguished from each other (Ellenberg et al. 1999; Yang et al. 1998).

We set out to develop stable broad-host-range vectors that drive expression of the enhanced cyan fluorescent protein (ECFP) and enhanced yellow fluorescent protein (EYFP). Our results show that various rhizobial species can be marked with these plasmids and the corresponding strains can be unambiguously identified during various stages of their symbiosis with a number of different plant species.

\section{RESULTS AND DISCUSSION}

Broad-host-range vectors driving expression of AFPs.

Initially, the coding sequence for EGFP was transferred into the broad-host-range vector pBBR1MCS-5 in frame with the LacZ- $\alpha$-peptide, which is expressed from the lac promoter. $E$. coli harboring such a plasmid (two clones, pMP2444 and pMP2463 were used) showed clear EGFP fluorescence when viewed by fluorescence microscopy using the appropriate excitation and emission filters. We noted that when Escherichia coli was grown under high selective pressure (gentamicin at $40 \mu \mathrm{g} / \mathrm{ml}$ instead of $10 \mu \mathrm{g} / \mathrm{ml}$ ) pMP2463 could be reproducibly mutated into a form that produced much higher levels of EGFP per cell than the unmutated form as detected by fluorescence microscopy. Analysis of plasmid DNA isolated from 
these cells suggested that this mutation strongly increased the plasmid copy number (data not shown). The same effect on expression was noted when one of the mutated isolates (pMP2464) was transferred to various rhizobia species, suggesting that the copy number effect is maintained across bacterial species.

To express other AFPs, the coding sequence for ECFP and EYFP was inserted into pBBR1MCS-5, resulting in pMP4516 and pMP4518, respectively. E. coli transformed with each plasmid indeed expressed the expected protein as determined by fluorescence microscopy using the appropriate filter sets. When crossed into various rhizobial species, cells became fluorescent with the expected excitation and emission characteristics. As a result, it was possible to mix two populations of rhizobia, each expressing a different fluorescent protein, and to trace the individual populations within the mixture. To specifically detect bacteria expressing ECFP or EYFP, we used a confocal laser scanning microscope that allows detection of emitted light at freely selectable wavelengths. For ECFP, objects were excited with light of $457 \mathrm{~nm}$ and emitted light was detected around $480 \mathrm{~nm}$, whereas for EYFP, excitation was at $488 \mathrm{~nm}$ and detection around $540 \mathrm{~nm}$. To avoid cross talk, the images for each channel were acquired separately.

Insertion of the ECFP and EYFP coding sequence into pMP2464 resulted in plasmids pMP4517 and pMP4519 (Fig. 1). Analysis of plasmid stability of these two plasmids in various rhizobial species (also discussed below) showed, surprisingly, that the high-copy, ECFP-containing derivative pMP4517 was less stable in the absence of antibiotics in species Mesorhizobium loti R7A and S. fredii USDA201. Al-

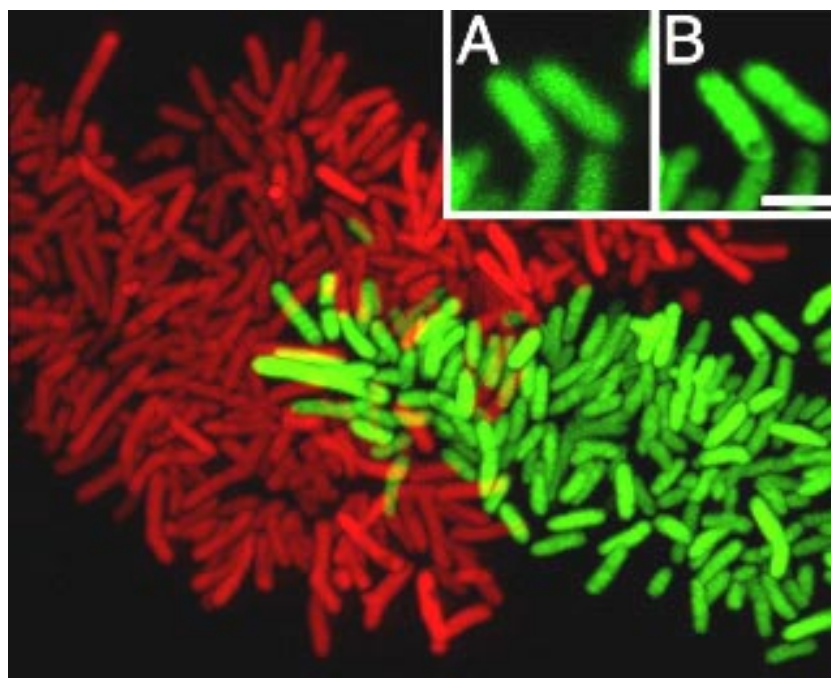

Fig. 1. Rhizobium leguminosarum bv. viciae RBL 5560 carrying pMP4517 and expressing enhanced cyan fluorescent protein (ECFP) (green) and the same strain transformed with pMP4519 and expressing enhanced yellow fluorescent protein (EYFP) (red). Cells growing on agar plates were resuspended in water, mixed, and placed between a coverslip and glass slide for microscopical observation. Optical sections were obtained with a Leica SP confocal microscope (Leica, Bensheim, Germany) using separate illumination at $457 \mathrm{~nm}$ (ECFP) or $488 \mathrm{~nm}$ (EYFP) and detection at 479 to $490 \mathrm{~nm}$ (ECFP) or 520 to $550 \mathrm{~nm}$ (EYFP). The image stack was deconvoluted with the Huygens software package, and a projection of all optical sections is shown. The apparent banding pattern in the bacteria is only seen when bacteria are located in different optical sections and is apparently an artifact of the projection method. A, Part of the main image before deconvolution. B, same field as in $\mathbf{A}$ after deconvolution. Size bars indicate Main figure, $5 \mu \mathrm{m}$ and $\mathbf{A}$ and $\mathbf{B}, 2 \mu \mathrm{m}$. though this effect was not clearly noticeable with the EYFPcontaining plasmid pMP4519, we decided not to use the highcopy-number derivatives for infection studies.

A second set of AFP-expressing vectors was constructed using plasmid pME6010, which is known to be very stable in various soil bacteria (Heeb et al. 2000). The resulting plasmids, pMP4657, pMP4656, and pMP4639, confer tetracycline resistance and express the EGFP, ECFP and EYFP genes, respectively, driven by the lac promoter.

The various constructs as well as the relative levels of AFP expression in rhizobia transfected with these plasmids are summarized in Figure 2. Results show that levels of fluorescence correlate with the presumed copy number of the plasmid replication origin.

\section{Detection of mixed populations of bacteria on plant roots.}

Next, it was of interest to determine whether two populations of bacteria could be detected on plant roots. We therefore inoculated vetch (Vicia sativa) with $R$. leguminosarum bv. viciae strain RBL5560, Lotus japonicus with M. loti R7A, alfalfa with $S$. meliloti 1021, and siratro (Macrophillium atropurpureum) with $S$. fredii $\mathrm{HH} 103$, as well as Rhizobium sp. strain NGR 234. Prior to inoculation, derivatives of the same strains carrying either pMP4516 or pMP4518 were mixed in ratios varying between 5:1 and 1:5. In each case, we were able to detect the two differently fluorescing bacteria on the plant roots (Fig. 3A-C), demonstrating that the progeny of the inoculated bacteria can be detected and discriminated from each other also when grown under nonselective conditions.

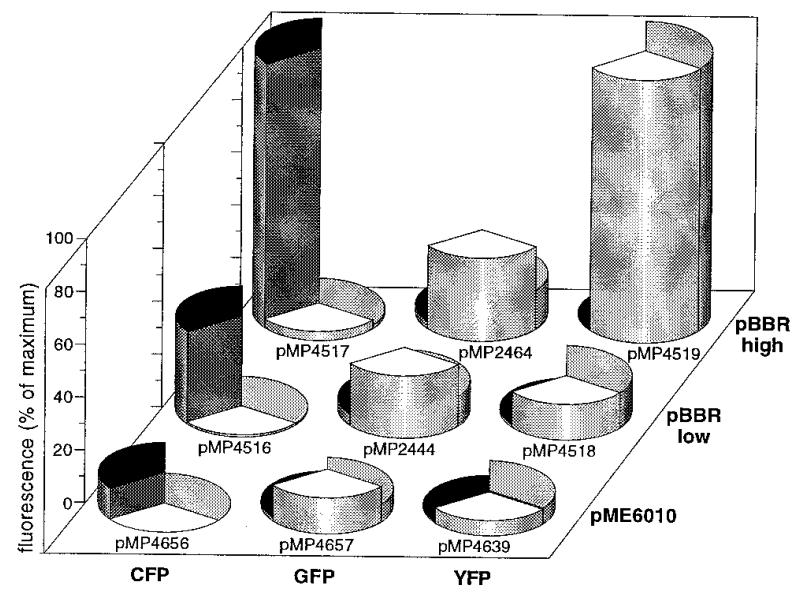

Fig. 2. Relative expression levels of autofluorescent proteins (AFPs) expressed on different plasmids. Expression plasmids based on different origins of replication (listed on the right) were made with different AFPs (bottom) and transconjugated into Rhizobium leguminosarum biovar viciae strain RBL5560. Cells (200 mg) growing on agar plates were suspended in $200 \mu \mathrm{l}$ of water. Fluorescence of cell suspensions was measured in a HTS7000 bioassay reader fluorometer (Perkin \& Elmer Life Sciences, Oosterhout, The Netherlands) equipped with filter sets for enhanced cyan fluorescent protein (ECFP) (black bars, excitation at $430 \mathrm{~nm}$ with emission at $485 \mathrm{~nm}$ ), enhanced green fluorescent protein (EGFP) (white bars, excitation at $485 \mathrm{~nm}$ with emission at $520 \mathrm{~nm}$ ), and enhanced yellow fluorescent protein (EYFP) (gray bars excitation at $485 \mathrm{~nm}$ with emission at $535 \mathrm{~nm}$ ). Fluorescence intensities are expressed as a percentage of the maximum value measured with each filter set (note that it is not meaningful to compare values measured with different filter sets). 


\section{Detection of mixed populations}

\section{of bacteria inside plant roots.}

In all cases, inoculation of plants with rhizobia harboring plasmids that drive expression of AFPs resulted in the development of nodules on the plant roots within 5 to 15 days, comparable to the time required for nodules to form on plants infected with rhizobia not producing AFPs. The entry of bacteria into the plant roots could also be visualized more directly; in many cases, infection threads with either ECFP- or EYFP-expressing bacteria were easily identified (Fig. 3D). In at least one case, we observed an infection thread that contained bacteria expressing ECFP as well as bacteria expressing EYFP (Fig. 3E). Interestingly, this infection thread seems to consist of multiple arrays (strings) of bacteria, with each array expressing the same AFP.

In many cases, fluorescent bacteria were found inside nodules. Examples are shown in Figure 4. Bacteroids expressing
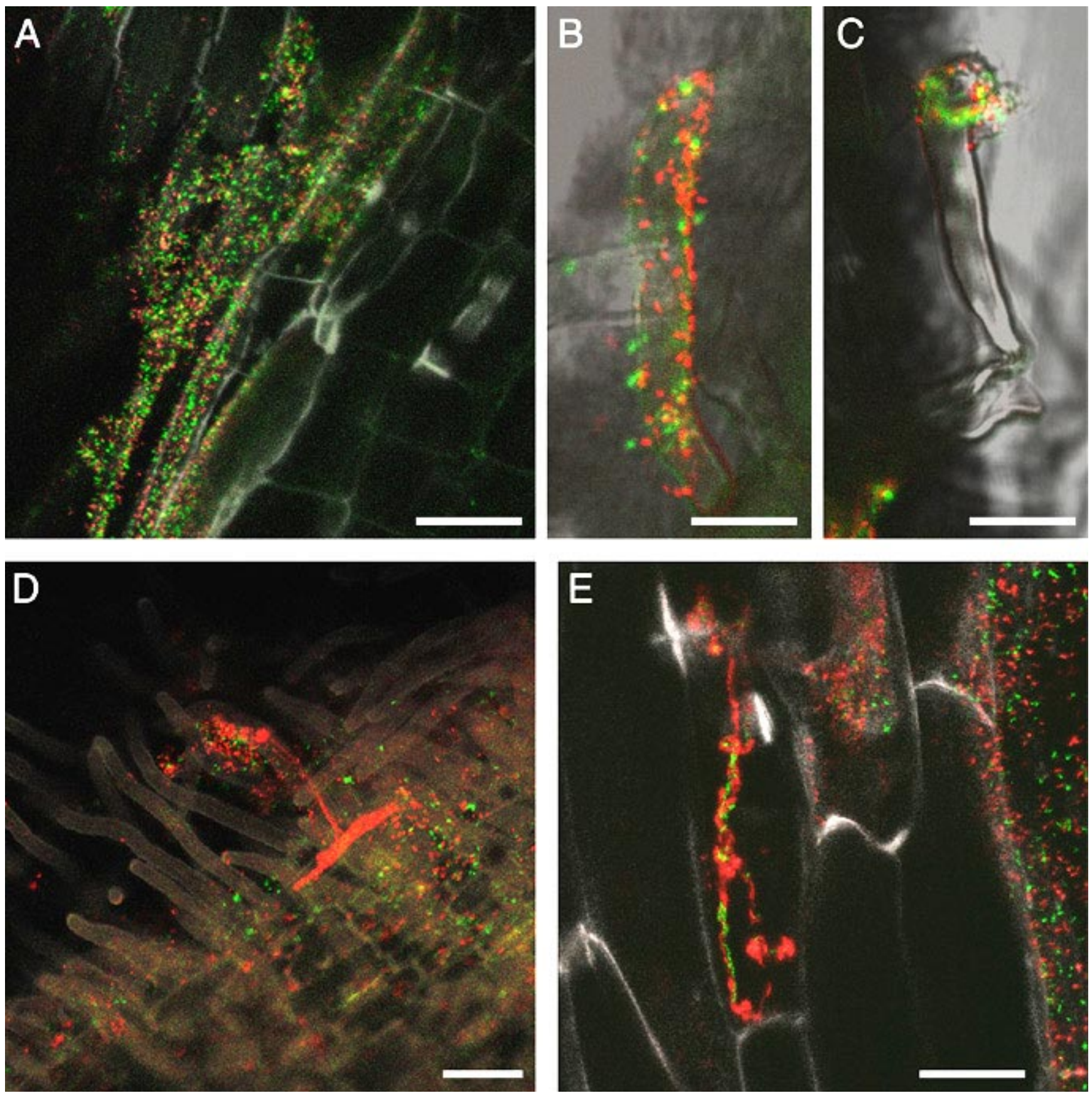

Fig. 3. Rhizobia expressing enhanced cyan fluorescent protein (ECFP) (as encoded on plasmid pMP4516) or enhanced yellow fluorescent protein (EYFP) (encoded on pMP4518) on and in plant roots. In all panels ECFP-expressing bacteria appear green, EYFP-expressing bacteria appear red, and the white/gray depicts an image obtained by measuring $\mathbf{A}, \mathbf{D}$, and $\mathbf{E}$, the reflected light or $\mathbf{B}$ and $\mathbf{C}$, transmitted light in bright field mode. Image acquisition was as described in Figure 1. A, Vetch root infected with Rhizobium leguminosarum bv. viciae RBL5560. B and C, Root hairs of Lotus japonicus infected with Mesorhizobium loti R7A. D, Root hair of vetch containing a fluorescent infection thread formed by R. leguminosarum bv. viciae RBL5560. E, Infection thread in the root cortex of vetch containing both $R$. leguminosarum bv. viciae expressing ECFP and the same strain expressing EYFP. Size bars represent $\mathbf{A}$ and $\mathbf{D}, 50 \mu \mathrm{m}$ or $\mathbf{B}, \mathbf{C}$, and $\mathbf{E}, 25 \mu \mathrm{m}$. 
ECFP were found in nodules formed by siratro infected with S. fredii HH103 carrying plasmid pMP4516 (Fig. 4A and B). These bacteroids could also be visualized by two-photon excitation at $810 \mathrm{~nm}$ (Fig. 4B). Two-photon excitation has several advantages over one-photon excitation, including the ability to image deeper into tissues (Piston 1999). Moreover, 810-nm light efficiently excites ECFP through two-photon absorption but barely excites EYFP, whereas the most widely available one-photon laser line for ECFP excitation (the 457-nm line from an argon laser) excites EYFP to a considerable extent (N. Stuurman, unpublished data). Mixtures of rhizobia expressing ECFP or EYFP were found in nodules from vetch infected with mixtures of $R$. leguminosarum bv. viciae RBL5560 containing plasmids pMP4516 or pMP4518 (Fig. 4C). To test whether different species of rhizobia can be traced back to the same nodule, we infected siratro with a mixture of Rhizobium sp. strain NGR 234 carrying plasmid pMP4516 and S. fredii HH103 transformed with pMP4518. As documented in Figure
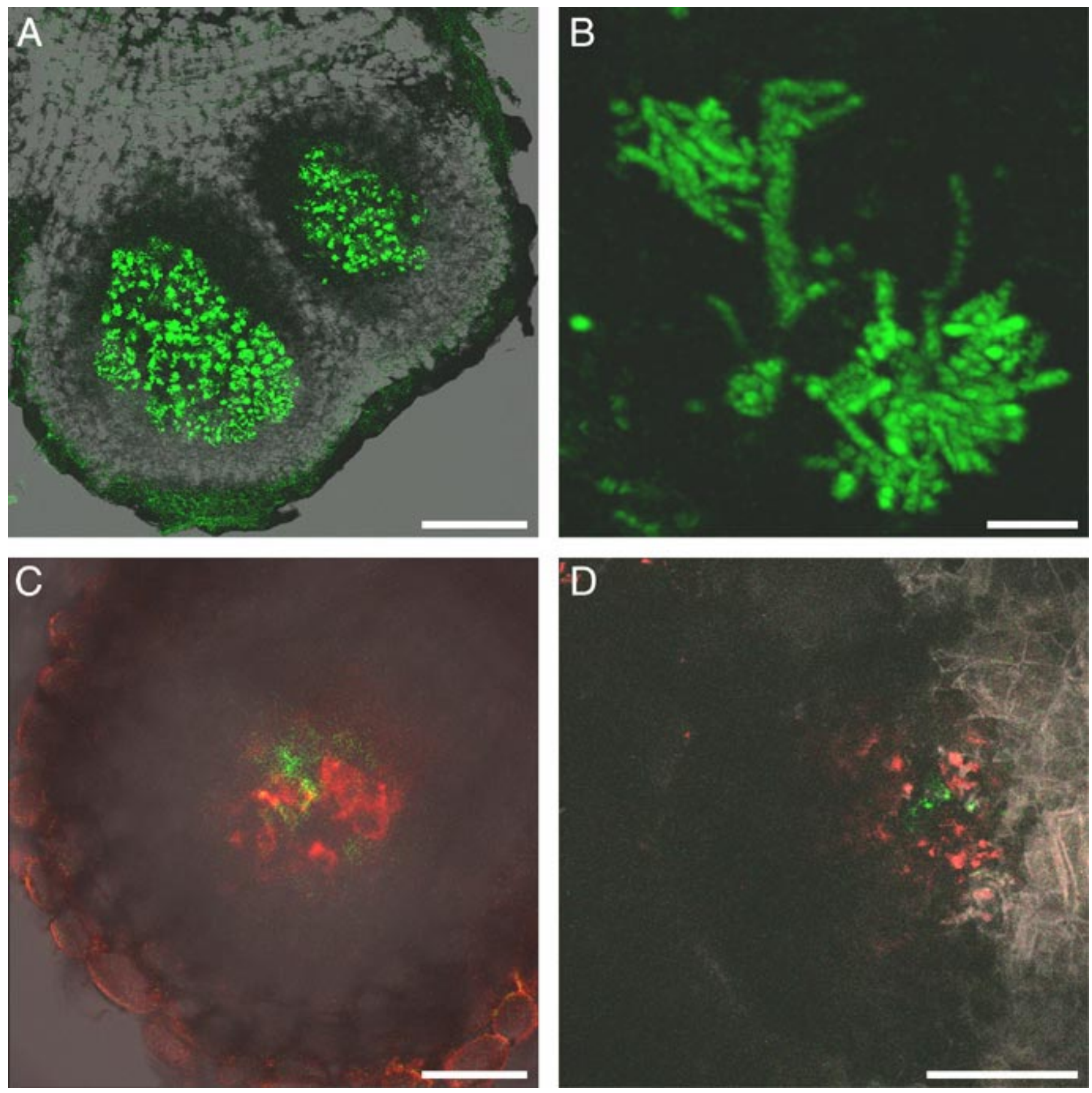

Fig. 4. Rhizobia expressing enhanced cyan fluorescent protein (ECFP) or enhanced yellow fluorescent protein (EYFP) in nodules. Colors represent objects as described in Figure 3. Image acquisition was as described in Figure 1 (except for B). A, Nodule of siratro infected with Sinorhizobium fredii strain HH103 carrying plasmid pMP4516 driving expression of ECFP. B, Same nodule as shown in A. Excitation was in this case with $810 \mathrm{~nm}$. The image was deconvoluted using a theoretically calculated point spread function. C, Nodule formed by vetch roots infected with a mixture of Rhizobium leguminosarum bv. viciae RBL5560 expressing ECFP and the same strain expressing EYFP. D, Part of a nodule of siratro infected with a mixture of Rhizobium sp. strain NGR234 expressing ECFP and $S$. fredii HH103 expressing EYFP. Size bars represent A, C, and D, $200 \mu \mathrm{m}$ or B, $2.5 \mu \mathrm{m}$. 
4D, bacteria expressing ECFP or EYFP were both found in the same nodule, demonstrating not only that this methodology enables fast and easy identification of rhizobial species in vivo, but also that more than one rhizobial species can be present in one and the same nodule. Previously, it has been shown that $R$. leguminosarum bv. trifolii can infect pea when inoculated together with $R$. leguminosarum bv. viciae and that both species can be reisolated from one nodule (Johnston and Beringer 1976).

\section{Plasmid stability.}

The stability of plasmids pMP2444 and pMP2464 in the absence of selection pressure was tested in the rhizobial species $R$. leguminosarum bv. viciae strains RBL5560 and 248, R. leguminosarum bv. trifolii strain ANU843, S. meliloti strain 2011, S. fredii strains USDA201 and USDA191, M. loti strains NZP2235 and R7A, Bradyrhizobium japonicum strain USDA110, and Asorhizobium caulinodans strain ORS571. No loss of plasmid was detected, as measured by green fluorescence of single colonies after four times of continuous restreaking on YMB agar plates, except for B. japonicum strain USDA110, which lost plasmid pMP2464 at a frequency of at least $50 \%$. We also analyzed whether the EGFP expressing plasmids were stable during symbiosis by reisolation of bacteria from root nodules of vetch that had been infected with $R$. leguminosarum bv. viciae strain RBL5560. Results showed that $86 \%$ of the reisolated bacteria carried plasmid pMP2444 and 60\% retained plasmid pMP2464.

Stability of the pME6010-derived plasmids (pMP4657, pMP4656, and pMP4639) was tested in R. leguminosarum bv. viciae strain RBL5560, M. loti strain R7A, and S. meliloti strain 2011. Results showed that these plasmids were completely maintained after four times of successive restreaking on nonselective medium. M. loti strain R7A and S. meliloti strain 2011, both containing plasmid pMP4639 (containing the EYFP coding sequence), were reisolated from root nodules of L. japonicus and Medicago sativa plants, respectively, and analyzed for fluorescence on nonselective medium. Results showed that these species maintained plasmid pMP4639 in $100 \%$ of the reisolated bacteria.

\section{Movements of bacteroids.}

While inspecting bacteroids in nodules, we noted that they often moved around in the cytoplasm of the infected plant cell. A detailed example is shown in Figure 5. Individual bacteroids showed complex movements with respect to each other. For instance, the bacteroid indicated with arrows in the insets of Figure 5 seems to move around in a semicircle, whereas most of the surrounding bacteroids do not show such movement. A few other bacteroids in the same cells moved at similar speeds. A movie displaying the complete time series of the whole image can be found at http://rulbim.leidenuniv.nl/publications/MPMI_Stuurman.htm. Very clearly, at this stage of infection, bacteroids are not static structures but rather display spatially dynamic behavior.

\section{MATERIALS AND METHODS}

\section{AFP expression vectors.}

To express AFPs on the broad-host-range vector pBBR1MCS5 , coding sequences were transferred from pEGFP-1 (Clon- tech, Palo Alto, CA, U.S.A.), pECFP (Clontech), and pEYFP (Clontech) using unique $\mathrm{Xba \textrm {I }}$ and $\mathrm{BamHI}$ restriction sites into similarly digested pBBR1MCS-5 (Kovach et al. 1995). The ensuing plasmids (pMP2444 and pMP2463 with EGFP, pMP4516 with ECFP, and pMP4518 with EYFP) produced the expected AFP in E. coli (strain 1164) as detected by fluorescence microscopy using the appropriate filter sets. We noted that mutants of pMP2463 expressing higher levels of EGFP easily arose in $E$. coli (discussed above). One of such mutants was isolated and called pMP2464. The higher levels of EGFP expression likely result from the enhanced copy number of pMP2464 as judged from gel-electrophoretic analysis of purified plasmid DNA. Transfer of pMP2444, pMP4516, and pMP4518 to various Rhizobium species (by triparental mating) yielded bacteria expressing the expected AFP as judged by fluorescence microscopy.
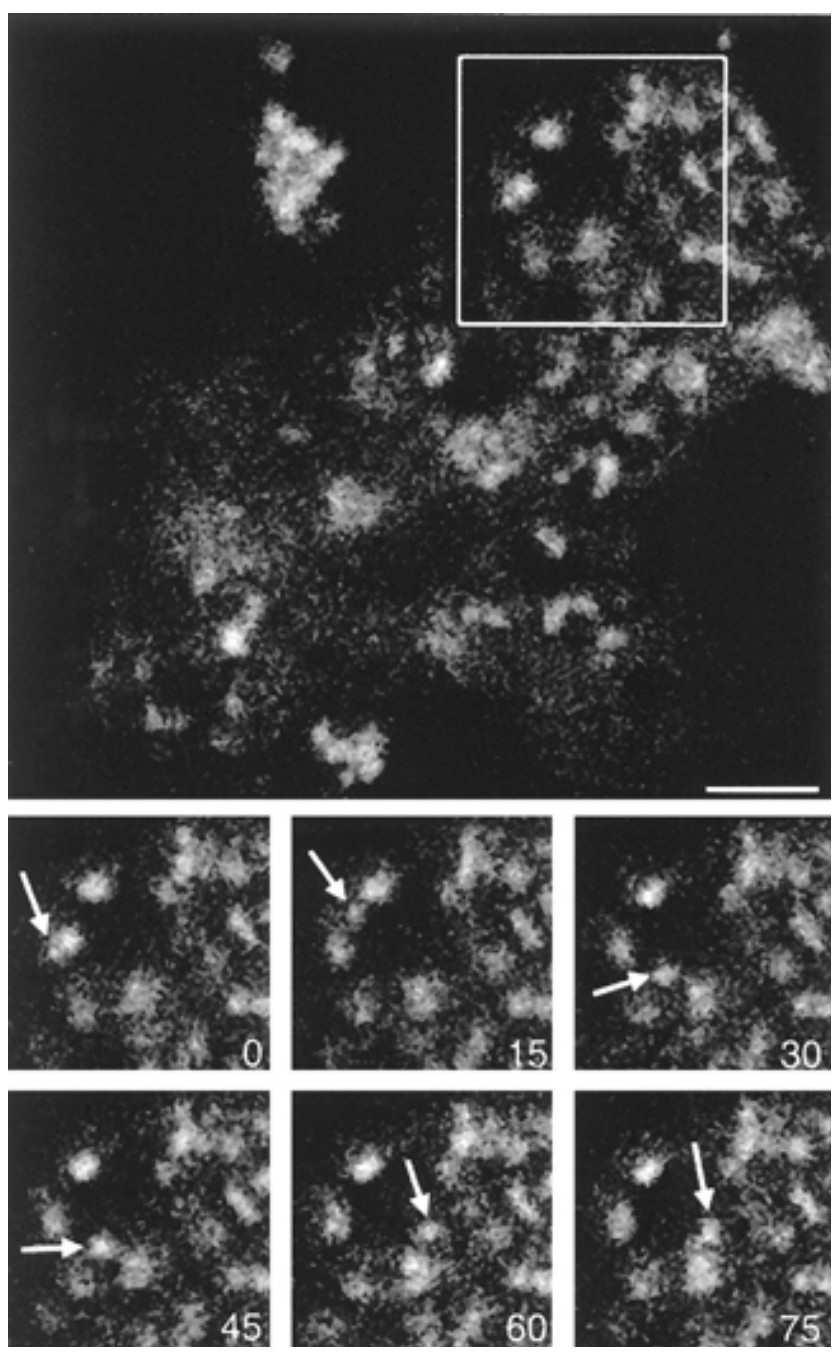

Fig. 5. Movements of bacteroids. All images were acquired from a 150$\mu \mathrm{m}$-thick microtome cut section from one nodule of Lotus japonicus infected with Mesorhizobium loti R7A transformed with plasmid pMP4518 expressing enhanced yellow fluorescent protein (EYFP). The insets show the area indicated with a white square in the main panel, each image in the insets was taken at the indicated time point $(0,15,30$, 45, 60, and $75 \mathrm{~s}$ ). Arrows point to what appears to be one and the same bacteroid rapidly moving with respect to the other bacteroids. Size bar represents $2.5 \mu \mathrm{m}$ and applies to all panels. 
To express AFPs in another stable replicon (that confers tetracycline resistance), the genes encoding EGFP, ECFP, and EYFP were inserted into plasmid pME6010 (Heeb et al. 2000). For the cloning procedure, we used 1.8-kb BglII-SacI restriction fragments derived from plasmids pMP2444, pMP4516, and pMP4518, respectively, containing the lac promoter as well as sequences coding for the fluorescent proteins. The resulting plasmids were named pMP4657 (EGFP), pMP4656 (ECFP), and pMP4639 (EYFP).

The stability of plasmids in the absence of antibiotics was tested by streaking out single colonies on yeast mannitol broth (YMB) agar plates (lacking antibiotics) for four successive times. Plasmid loss was scored by counting the number of colonies that were no longer green fluorescent or that displayed sectors with diminished fluorescence. Reisolation of bacteria from 6-week-old nodules from vetch and $L$. japonicus was performed as described previously (Vincent 1970).

\section{Inoculation of plants.}

Seeds of vetch, L. japonicus, Medicago sativa, and siratro were surface-sterilized and germinated as described previously (Pacios Bras et al. 2000). Germinated seeds were placed on agar slants made with nitrogen-free Jensen medium and inoculated with the appropriate rhizobial species. Plant roots were shielded from light. Plants grew at $20^{\circ} \mathrm{C}$ (vetch, L. japonicus) or at $23^{\circ} \mathrm{C}$ (siratro) using a 16-h light/8-h dark regime.

\section{Microscopy.}

Bacterial colonies were examined using a Leica MZFLIII stereo microscope equipped with epifluorescence detection (Leica, Bensheim, Germany). Filter sets tailored to the specific chromophores were used (for ECFP, 440/21-nm excitation with 480/36-nm emission; for EGFP, 480/40-nm excitation with 510-nm long pass emission; and for EYFP, 510/20-nm excitation with 560/40-nm emission).

Plant roots inoculated with bacteria were mounted in deionized water on a coverslip attached to homemade holders. In some cases, $150-\mu \mathrm{m}$-thick sections were prepared using a Leica VT1000S microtome. Samples were examined with an inverted fluorescence microscope (Leica-DMIRBE) equipped with filter blocks with spectral properties matching those of ECFP (440/21-nm excitation with 480/36-nm emission, XF114; Chroma, Brattleboro, VT, U.S.A.) or EGFP and EYFP (470/20-nm excitation with 515-nm long pass emission, I3; Leica). A Leica SP confocal scanhead was also attached to this microscope. Usually, dual color images were acquired by sequentially scanning with settings optimal for ECFP (excitation with the 457-nm Argon laser line, emission detection between 470 and $490 \mathrm{~nm}$ ), followed by settings optimal for EYFP (excitation with the 488-nm Argon laser line, detection of emitted light between 530 and $550 \mathrm{~nm}$ ). Cross talk between the channels in this setup was always monitored and, in all cases, negligible. Often, we also acquired images by measuring the laser light reflected by the object (reflected light images) or a bright field image using a photo-multiplier detector measuring the transmitted light. In some cases, ECFP was excited using a TiS Mira 900F laser (Coherent Inc., Sunnyvale, CA, U.S.A.) tuned to emit light at $810 \mathrm{~nm}$. The laser light was run through a Mira GDC unit (Coherent Inc.), and fiber-coupled into the microscope, resulting in pulse lengths of around $10 \mathrm{ps}$ at an average power of about $300 \mathrm{~mW}$.
For time-lapse microscopy, the confocal microscope was programmed to acquire an optical section (at the same plane) every $15 \mathrm{~s}$.

To represent 3-D digital data, most often projections were made from the individual channels in the image stacks using the software package Imaris (Bitplane A.G., Zürich, Switzerland) or Scion Image (Scion Corporation, Frederick, MD, U.S.A.) enhanced with self-written macros. The projections of the individual channels were merged in Photoshop 5.0 (Adobe, San Jose, CA, U.S.A.), and intensities of the individual channels were adjusted to facilitate visualization. Whenever comparison between different images was important, care was taken to process the images identically.

In some cases, the resolution of the original data was enhanced by subjecting them to a deconvolution procedure using the maximum likelihood estimate method as offered by the Huygens software package (SVI, Hilversum, The Netherlands). For images acquired with one-photon excitation, we used point spread functions as measured on our microscope according to the instructions provided with the Huygens package, whereas for images acquired with two-photon excitation, we used theoretically determined point spread functions. Representation of deconvoluted images was as described above for the raw data.

\section{ACKNOWLEDGMENTS}

Part of this work was supported by the EC INCO-DC project "Improvement of symbiotic nitrogen fixation in Chinese soybean cropping areas" contract number ERBIC18CT970191. We thank Stephan Heeb and Dieter Haas for kindly providing the cloning vector pME6010 and Peter Hock for his help in preparing figures.

\section{LITERATURE CITED}

Chalfie, M., Tu, Y., Euskirchen, G., Ward, W. W., and Prasher, D. C. 1994. Green fluorescent protein as a marker for gene expression. Science 263:802-805.

Cheng, H. P., and Walker, G. C. 1998. Succinoglycan is required for initiation and elongation of infection threads during nodulation of alfalfa by Rhizobium meliloti. J. Bacteriol. 180:5183-5191.

Ellenberg, J., Lippincott, S. J., and Presley, J. F. 1999. Dual-colour imaging with GFP variants. Trends Cell Biol. 9:52-56.

Gage, D. J., Bobo, T., and Long, S. R. 1996. Use of green fluorescent protein to visualize the early events of symbiosis between Rhizobium meliloti and alfalfa (Medicago sativa). J. Bacteriol. 178:71597166.

Hadri, A.-E., Spaink, H. P., Bisseling, T., and Brewin, N. J. 1998. Diversity of root nodulation and rhizobial infection processes. Pages 347-360 in: The Rhizobiaceae. H. P. Spaink, A. Kondorosi, and P. J. J. Hooykaas, eds. Kluwer Academic Publishers, Dordrecht, The Netherlands.

Heeb, S., Itoh, Y., Nishijyo, T., Schnider, U., Keel, C., Wade, J., Walsh, U., O'Gara, F., and Haas, D. 2000. Small, stable shuttle vectors based on the minimal pVS1 replicon for use in gramnegative plant-associated bacteria. Mol. Plant-Microbe Interact. 13: 232-237.

Johnston, A. W. B., and Beringer, J. E. 1976. Pea root nodules containing more than one Rhizobium species. Nature 263:502-504.

Kovach, M. E., Elzer, P. H., Hill, D. S., Robertson, G. T., Farris, M. A., Roop, R. M., and Peterson, K. M. 1995. Four new derivatives of the broad-host-range cloning vector pBBR1MCS, carrying different antibiotic-resistance cassettes. Gene 166:175-176.

Matus, A. 1999. GFP in motion CD-ROM-Introduction: GFP illuminates everything. Trends Cell Biol. 9:43.

Pacios Bras, C., Alberich Jordá, M., Wijfjes, A. H. M., Harteveld, M., Stuurman, N., Thomas-Oates, J. E., and Spaink, H. P. 2000. A Lotus 
japonicus nodulation system based on heterologous expression of the fucosyl transferase NodZ and the acetyl transferase NolL in Rhizobium leguminosarum. Mol. Plant-Microbe Interact. 13:475479.

Piston, D. W. 1999. Imaging living cells and tissues by two-photon excitation microscopy. Trends Cell Biol. 9:66-69.

Pueppke, S. G., and Broughton, W. J. 1999. Rhizobium sp. strain NGR234 and $R$. fredii USDA257 share exceptionally broad, nested host ranges. Mol. Plant-Microbe Interact. 12:293-318.

Tsien, R. Y. 1998. The green fluorescent protein. Annu. Rev. Biochem. 67:509-544.
Vincent, J. M. 1970. A Manual for the Practical Study of the RootNodule Bacteria. Blackwell Scientific Publications, Oxford.

Xi, C., Lambrecht, M., Vanderleyden, J., and Michiels, J. 1999. Bifunctional $g f p$ - and $g u s A$-containing mini-Tn 5 transposon derivatives for combined gene expression and bacterial localization studies. J. Microbiol. Methods 35:85-92.

Yang, T. T., Sinai, P., Green, G., Kitts, P. A., Chen, Y. T., Lybarger, L., Chervenak, R., Patterson, G. H., Piston, D. W., and Kain, S. R. 1998. Improved fluorescence and dual color detection with enhanced blue and green variants of the green fluorescent protein. J. Biol. Chem. 273: 8212-8216. 